\title{
Rooted Spiral Trees on Hyper-Cubic Lattices
}

\author{
Sumedha $^{1}$ \\ Received October 15, 2004; accepted April 8, 2005
}

\begin{abstract}
We study rooted spiral trees in 2,3 and 4 dimensions on a hyper cubic lattice using exact enumeration and Monte-Carlo techniques. On the square lattice, we also obtain exact lower bound of 1.93565 on the growth constant $\lambda$. Series expansions give $\theta=-1.3667 \pm 0.0010$ and $v=0.6574 \pm 0.0010$ on a square lattice. With Monte-Carlo simulations we get the estimates as $\theta=-1.364 \pm 0.010$, and $v=0.656 \pm 0.010$. These results are numerical evidence against earlier proposed dimensional reduction by four in this problem. In dimensions higher than two, the spiral constraint can be implemented in two ways. In either case, our series expansion results do not support the proposed dimensional reduction.
\end{abstract}

KEY WORDS: Spiral trees; exact enumeration; dimensional reduction.

\section{INTRODUCTION}

Spiral structures are very common in nature. Some examples of the beautiful spiral structures in galaxies, shoot arrangement in plants, polymers with spiral structure etc may be found in the book by Hargittai. ${ }^{(1)}$ In statistical mechanics, lattice models of spiral self avoiding walks have been studied and can be solved exactly in two dimension, ${ }^{(2,3)}$ though no solution is known for the self avoiding walks without the spiral constraint. A model of spiral trees and animals was proposed by Li and Zhou, ${ }^{(4)}$ which based on numerical studies was suggested to be in a new universality class. This problem was further studied by Bose et al. ${ }^{(5)}$ Based on the numerical evidence, and guided by the fact that magnetic field acting perpendicular to the motion of a charged particle produces spiralling motion and reduction by two in effective dimensionality, they conjectured that spiral tree

\footnotetext{
${ }^{1}$ Department of Theoretical Physics, Tata Institute of Fundamental Research, Homi Bhabha Road, Mumbai 400005, India; e-mail: sumedha@theory.tifr.res.in
}

\section{OF1}


problem would show a dimensional reduction by four. They conjectured the exponents of the spiral tree problem follow the following relations

$$
\begin{array}{cc}
\theta=(d-4) v_{p l} & \text { for } d=2 \\
\theta=(d-4) v_{\perp} & \text { for } d>2
\end{array}
$$

where $\theta$ is the entropic exponent and $v_{p l}$ and $v_{\perp}$ are the exponents related to the radius of gyration in the plane in which the tree has a rotational constraint and perpendicular to that plane, respectively.

Since then this problem has not been studied further. Dimensional reduction is an intriguing possibility. The lattice tree model without spiral constraint is known to show a dimensional reduction by two. ${ }^{(6)}$ The directed version, show a dimensional reduction by one. For both models, the tree and animals are believed to lie in the same universality class. In this paper, we revisit the problem and obtain a significantly longer series for rooted spiral trees. Specifically in two dimensions we have added twelve terms to the earlier series of 25 terms. In three and four dimensions, we generated a seventeen and a thirteen term series, respectively. The earlier known series in three and four dimensions had thirteen and nine terms. In the process, we also correct some mistakes in the earlier reported series. We also perform Monte-Carlo (MC) simulations using the improved incomplete enumeration algorithm ${ }^{(7)}$ and generate spiral trees up to sizes of 1000 in two dimensions. Our analysis of exact series and MC samples do not support the conjectured dimensional reduction by four in this problem.

A lattice tree is a cluster of connected sites which contains no loops. Spiral trees are a subclass of lattice trees. In a tree every cluster site is attached to the origin through a unique path. In a spiral tree, this path has a specific rotational constraint.

We define rooted spiral tree as a acyclic connected subgraph of a lattice such that the projection of the path joining any site of the tree to the root on $x-y$ plane contains no left turn (Fig. 1). We will measure the size of a spiral tree by the number of sites present in the tree. These are called spiral site trees. The number of possibilities of spiral bond trees are more than that for spiral site trees but both are thought to lie in the same universality class. For example, the site marked as $X$ in Fig. 1 is not allowed in spiral site tree as it would introduce a loop. But it can be present in a spiral bond tree. Let the total number of distinct rooted spiral trees be $A_{n}$. This is expected to have a asymptotic behaviour of the form

$$
A_{n} \sim C \lambda^{n} n^{-\theta}
$$




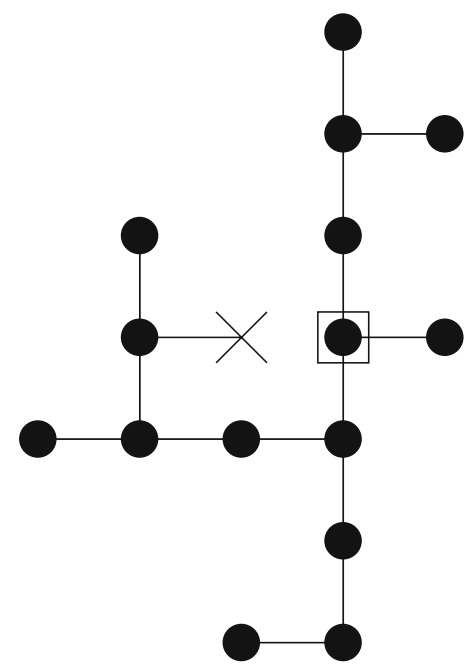

Fig. 1. A rooted spiral tree of 15 sites on a square lattice. The root is the site enclosed in the square. At the root site the tree has freedom of choosing any of the four neighbouring sites. We count the spiral tree by number of sites and hence all bonds between two occupied sites is always assumed to be present. The site marked by $X$, if present will result in a loop for spiral site trees and hence will not be allowed. But it can be present in a spiral bond tree.

where $C$ is a constant, $\theta$ is a critical exponent and is expected to depend only on the dimension of the lattice and $\lambda$ is known as the lattice dependent growth constant. The existence of growth constant $\lambda$ for unrooted lattice trees and animals has been proved rigorously using concatenation and super multiplicity arguments. ${ }^{(8)}$ Also a rigorous lower bound for $\theta$ for unrooted lattice trees and animals has been proved $^{(9)}$ using pattern theorem. Specifically, it is $\theta \geqslant(d-1) / d$, for any dimension $d \geqslant 2$. Equation 3 is expected to hold for most cluster enumeration problems on regular lattices, though other asymptotic forms are also possible. For example, for spiral self-avoiding walks on square and triangular lattices, $A_{n}$ tends to a stretched exponential in $n$ in the asymptotic limit. ${ }^{(2,3)}$ Though we do not prove existence of $\lambda$ and $\theta$ for spiral trees in this paper, we derive a lower bound for $\lambda$. Also, since spiral trees are a subset of lattice trees, $\lambda_{\text {spiral }} \leqslant \lambda_{\text {all }}$, where $\lambda_{\text {all }}$ is the growth constant for all trees, $\lambda_{\text {all }} \approx 3.795$ on a square lattice. ${ }^{(10)}$ In two dimensions, we have derived the generating function for enumeration of a subset of all possible spiral trees. The value of growth constant for this subset is 1.93565. This gives a lower bound on the growth constant $\lambda_{\text {spiral }}$ of the spiral trees on a square lattice. This bound will be derived in Section 2.1. 
For the conventional lattice animals, one can prove $\theta \geqslant 0$ through concatenation and super-multiplicity arguments. ${ }^{(8,9)}$ Concatenation does not work for spiral trees. Interestingly, our numerical studies give a negative value of $\theta$ in two and three dimensions.

The spiral trees are anisotropic. We measure the average extent of a $n$-site spiral tree in $x-y$ plane and perpendicular to the $x-y$ plane through the moment of inertia tensors, $I_{p l, n}$ and $I_{\perp, n}$, respectively. In the asymptotic limit, they are expected to vary as

$$
I_{p l, n} \sim A n^{2 v_{p l}+1}
$$

and

$$
I_{\perp, n} \sim A n^{2 v_{\perp}+1}
$$

where $v_{p l}$ and $v_{\perp}$ define the length scale of the spiral tree in planar and perpendicular direction, respectively.

\section{TWO-DIMENSIONAL LATTICE SPIRAL TREES}

Some pictures of randomly generated large spiral trees are shown in Fig. 2 (details later). One notes very long one-dimensional structures with infrequent turns. Hence, simple counting of structures of kind shown in Fig. 3 should give a good estimate of the growth constant $\lambda$. The generating function of trees of this type is easy to determine. If $A_{1}(x)$ is the generating function, we get

$$
A_{1}(x)=\frac{x}{1-x}+\frac{x^{3}}{(1-x)^{2}} A_{1}(x)
$$

which gives $A_{1}(x)=\frac{x(1-x)}{1+x^{2}-2 x-x^{3}}$. The number of trees of this type grows as $\lambda_{1}{ }^{n}$, with $\lambda_{1}=1.754878$. It is straightforward to include more complicated branches in such counting to get a better lower bound. This we proceed to do below.

\subsection{Lower Bound on Growth Constant on Square lattice}

Consider a subset of all the spiral trees on a square lattice rooted at the origin, which lie strictly in the first quadrant $x \geqslant 0, y \geqslant 0$; starting at the origin, and not touching $y=0$ and $y=1$ except at points $(0,0)$ and $(0,1)$, respectively. If $Q(x)$ is the generating function for spiral trees in a quadrant and if $q_{4, n}$ is the coefficient of $x^{n}$ in the expansion of $\left([Q(x)]^{4}\right) / x^{3}$, 

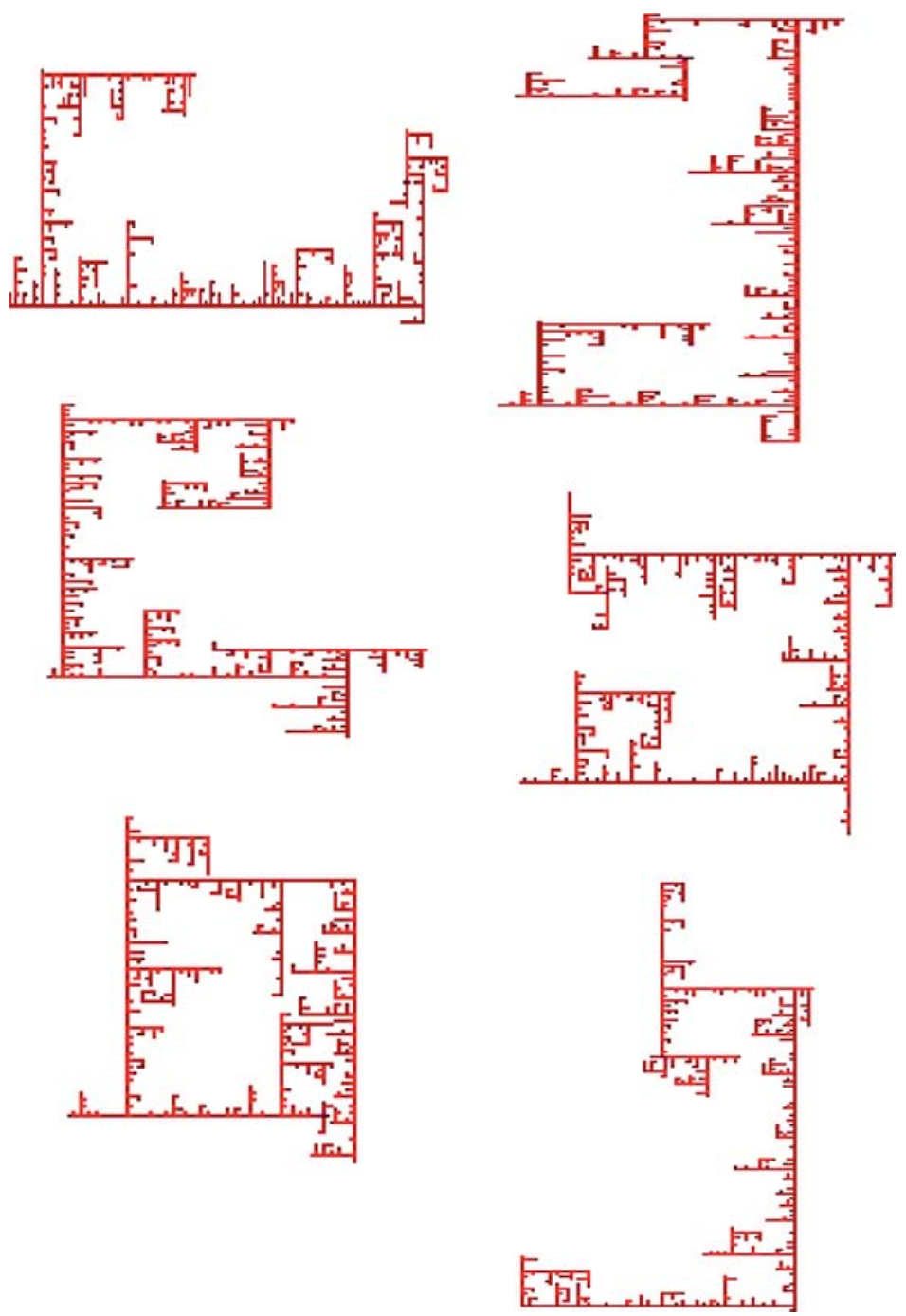

Fig. 2. Randomly generated spiral trees of 1000 sites in 2-dimensions using incompleteenumeration algorithm.

then

$$
A_{n} \geqslant q_{4, n}
$$

where $A_{n}$ is the $n^{\text {th }}$ term of $A(x)$, the generating function of all spiral trees on the square lattice. 


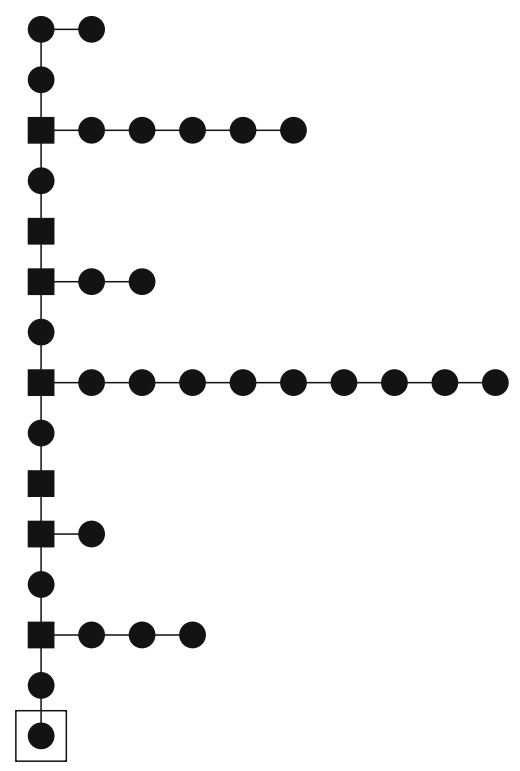

Fig. 3. A simple counting problem of backbone with arbitrary long offshoots. Minimum distance between two offshoots is 2 as else the tree constraint is violated. Solid squares represent the articulation points of the graph.

The enumeration of graphs contributing to $Q(x)$ can be made easier by noticing that these graphs can be formed by combination of smaller graphs. We define an articulation point ${ }^{(11)}$ as a point on $y$-axis such that the tree above is an allowed spiral tree in the quadrant above that part (note that these trees are defined in the upper quadrant and they never touch $y=0$ axis, except at $(0,0))$. For example, the solid squares represent the articulation points of the graph in Fig. 3, and Fig. 4 shows a spiral tree with no articulation point. Hence, these spiral trees can be seen as trees having $y$ axis as a backbone on which spiral graphs are connected at different articulation points maintaining the spiral constraint.

Let $B(x)$ be the generating function of the quadrant spiral trees with no articulation points. Hence $B(x)$ can be seen as sum of generating function of irreducible graphs with $i$ sites along $y$-axis. We represent them by $B_{i}(x)$ (see Fig. 5), then $B(x)=\sum_{i=1}^{\infty} B_{i}(x)$. The full generating function in terms of $B(x)$ would be

$$
Q(x)=x\left(1+B(x)+B^{2}(x) \ldots \ldots\right)=\frac{x}{1-B(x)}
$$




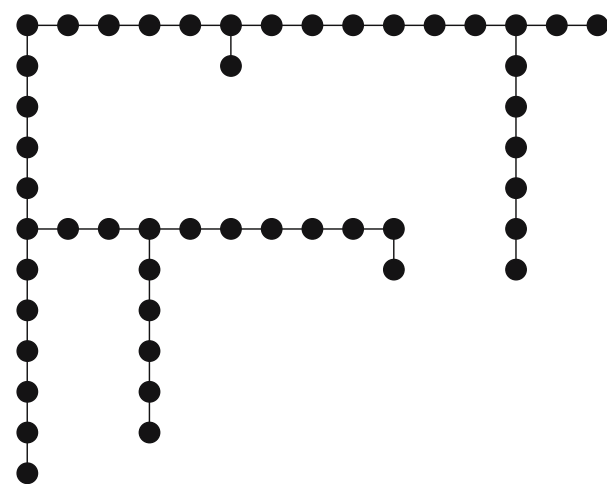

Fig. 4. An example of an irreducible spiral graph with no articulation point. This is also an example of a graph not included in $Q_{1}(x)$.

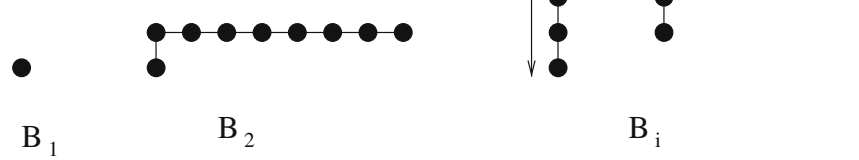

Fig. 5. Schematic figure of spiral trees contributing to $B_{i}(x), B_{1}(x)$ is just a single vertex.

where $B_{i}(x)$ are spiral graphs starting with $i$-sites along the $y$-axis. It is easy to see that $B_{1}(x)=x, B_{2}(x)=\frac{x^{3}}{1-x}$ and $B_{3}(x)=\frac{x^{6}}{\left(1-x-x^{3}\right)(1-x)}$. One can write $B_{4}(x)$ with some effort but we do not have a general form for $B_{i}(x)$ for any $i$.

We restrict the graphs contributing to $B_{i}(x)$ to be graphs such that they have $i$ sites along the $y$ axis and have at least one downward branch with $i-1$ sites. This would not include structures like Fig. 4. We will represent the generating function of these graphs by $Q_{1}(x)$. Then we can represent $B_{i}(x)$ in terms of two other generating functions, $V_{i}(x)$ and $W_{i}(x)$. We define $V_{i}(x)$ as the generating function of spiral subgraphs starting with having $i$ sites along $y$-axis. $W_{i}(x)$ is the generating function of spiral subgraphs starting with $i$-sites along $y$-axis and ending with a downward branch with $i-1$ sites (Fig. 6). Then,

$$
B_{i}(x)=W_{i}(x)+\frac{W_{i}(x) V_{i-1}(x)}{x^{i-1}}
$$



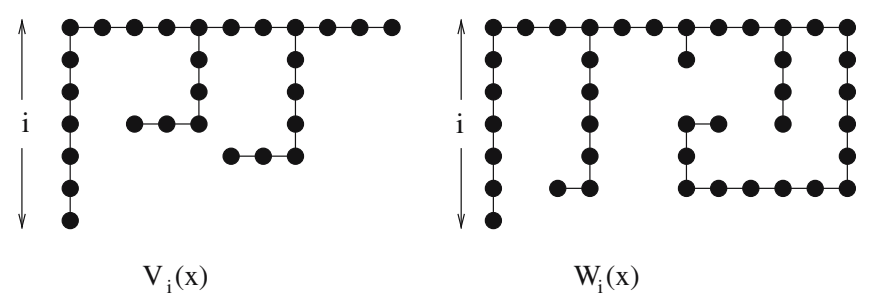

Fig. 6. Example of graphs contributing to $V_{i}(x)$ and $W_{i}(x)$, respectively.

Also, $V_{i}(x)$ can be rewritten in terms of $W_{i}(x)$ as

$$
V_{i}(x)=x V_{i-1}(x)+W_{i}(x)+\frac{W_{i}(x) V_{i-1}(x)}{x^{i-1}}
$$

By expressing $Q_{1}(x)$ in terms of $B_{i}(x)$ and $B_{i}(x)$ in turn in terms of $W_{i}(x)$, we can reduce the computational time. If $W_{n}$ is the number of graphs of size $n$ contributing to $W(x)\left(W(x)=\sum_{i=1}^{\infty} W_{i}(x)\right)$, and $Q_{n}$ is the number of graphs of size $n$ contributing to $Q_{1}(x)$, then $W_{n}$ grows more slowly than $Q_{n}$. We enumerated $W_{n}$ and using them we could generate a 56 term series for $Q_{1}(x)$. The computation time for $W_{n}$ grows as $(1.8)^{n}$, in contrast to $(2.04)^{n}$ for the $Q_{n}$ series.

If we restrict the graphs contributing to $B_{i}(x), W_{i}(x)$ and $V_{i}(x)$ to the graphs having comb-like structure (by comb-like structure we mean graphs with one-dimensional backbone having vertical straight lines of arbitrary lengths), then it turns out that one can get the exact expression for these generating functions. We represent them by $\widetilde{V}_{i}(x), \widetilde{W}_{i}(x)$ and $\widetilde{B}_{i}(x)$. It is easy to see that for comb like structures,

$$
W_{i}(x) \geqslant \widetilde{W}_{i}(x)=\frac{x^{2 i}}{1-x}+\frac{x^{2 i}}{1-x} \frac{K(x)}{1-x}+\frac{x^{2 i}}{1-x}\left(\frac{K(x)}{1-x}\right)^{2}+\cdots
$$

where $K(x)=x^{2} \sum_{j=1}^{i-2} x^{j}$. Hence,

$$
\widetilde{W}_{i}(x)=\frac{x^{2 i}(1-x)}{1-2 x+x^{2}-x^{3}+x^{i+1}}
$$

Similarly, we get

$$
\widetilde{V}_{i}(x)=\frac{x^{i+1}\left(1-x+x^{2}-x^{i}\right)}{1-2 x+x^{2}-x^{3}+x^{i+1}}
$$


and hence

$$
B_{i}(x) \geqslant \widetilde{B}_{i}(x)=\frac{x^{2 i}(1-x)^{2}}{\left(1-2 x+x^{2}-x^{3}+x^{i}\right)\left(1-2 x+x^{2}-x^{3}+x^{i+1}\right)}
$$

Substituting in Eq. (9) we get the generating function, $\widetilde{Q}_{1}(x)$ for this subset of spiral trees in a quadrant. This generating function has a singularity at $x_{c}=0.51662$ which gives the growth constant $\lambda^{\prime}$ of these trees to be 1.93565 . Since this counts only a subset of all the spiral trees on a square lattice, this is a rigorous lower bound on $\lambda_{\text {spiral }}$ for spiral trees on a square lattice.

For the full $Q_{1}(x)$, we derived a 56 term series. If we assume,

$$
Q_{n} \sim \lambda_{1}^{n} n^{-\theta_{1}}
$$

then we got estimates of $\lambda_{1}$ and $\theta_{1}$ to be

$$
\begin{aligned}
& \lambda_{1}=2.0449 \pm 0.0001 \\
& \theta_{1}=0.830 \pm 0.01
\end{aligned}
$$

\subsection{Exact Enumeration}

Since the number of configurations of a given cluster size is exponential in cluster size, the computational complexity of the algorithm for enumeration of all lattice animals or trees grows exponentially with the cluster size. For direct enumeration algorithms like Martin's algorithm, ${ }^{(12)}$ the time required to generate all the configurations of a given size grows as $\lambda^{n}$, where $\lambda$ is the growth constant and $n$ is the cluster size and the memory requirement grows like a polynomial in cluster size. For lattice trees and animals, a finite lattice method ${ }^{(13)}$ with an associated transfer matrix algorithm was used by Conway. (14) Conway generated a 25 term series for lattice animals using this algorithm. This series has recently been extended to 46 terms by Jensen ${ }^{(10)}$ with a slight improvement in the algorithm. Both space and time requirements of this algorithm are found numerically to approximately grow as $1.4^{n}$. The growth constant of lattice animals in contrast is approximately 4.06. Hence a considerable improvement in time is obtained by the transfer matrix algorithm at the cost of memory.

The spiral constraint, cannot be easily implemented using the transfer matrix. Hence we have used Martin's algorithm for spiral trees, making use 
of the fourfold rotational symmetry of the lattice. Our series for number of trees and their average moment of inertia is given in Appendix A.

Using this we generated a series of spiral trees on square lattice up to 37 terms (Appendix A). Earlier known series had only 25 terms.

For analysing the series data we tried a four parameter sequential fit to the data of the form

$$
A_{n}=B \lambda^{n}(n+\delta)^{-\theta}
$$

where $\delta$ is an adjustable fixed parameter and $B$ is a constant. We did a linear fit on the logarithm of Eq. (18) using $A_{n}, A_{n+1}, A_{n+2}$ and $A_{n+3}$ to estimate values of $B_{n}, \delta_{n}, \lambda_{n}$ and $\theta_{n}$. For spiral trees on square lattice we found a good convergence in successive values of $\lambda_{n}$ and $\theta_{n}$ for $\delta$ lying between 2.03 and 2.04. Fixing $\delta=2.0367$ and $B=0.18124$ we get a very good convergence of $\lambda_{n}$ and $\theta_{n}$ for different values of $n$. These are given in Appendix B. From this we estimate

$$
\begin{gathered}
\lambda=2.11433 \pm 0.00010 \\
\theta=-1.3667 \pm 0.0010
\end{gathered}
$$

We have tried fits with non analytic corrections to scaling of the form, $B \lambda^{n}(n+\delta)^{-\theta}\left[1+a / n^{\Delta}\right]$, but we didn't get good convergence for $\Delta$. Instead, $B \lambda^{n}(n+\delta)^{-\theta}\left[1-\alpha e^{-\beta n}\right]$ seems to fit much better with $\alpha \approx 0.32$ and $\beta \approx 0.35$.

For the radius of gyration data we used a sequential fit of the form

$$
\log I_{i, n}=\left(2 v_{i}+1\right) \ln (n+\delta)+u+\frac{v}{(n+\delta)^{2}}
$$

where $i$ stands for $p l$ or $\perp$ as the case maybe and $u$ and $v$ are constants.

For spiral trees in a plane $I_{\perp, n}$ would be zero and by symmetry the sum of square of $x$ coordinate of all sites for all configurations of clusters of size $n$ is symmetric with sum of squares of $y$-coordinate. Using Eq. (21) for sequential fit to our 35 term series we get a good convergence for $\delta$ lying between -0.33 and -0.35 . Fixing $\delta=-0.338$ we get the estimates of $v_{p l}$ to be

$$
2 v_{p l}=1.3148 \pm 0.0010
$$


Table I. Estimates of Critical Exponents and Growth Constant from Differential Approximants

\begin{tabular}{cccccc}
\hline$[l, m]$ & $x_{c}=1 / \lambda$ & $\theta$ & {$[l, m]$} & $x_{c}=1 / \lambda$ & $\theta$ \\
\hline$[14,13]$ & 0.47288256 & -1.36083 & {$[15,18]$} & 0.47307144 & -1.39078 \\
{$[14,14]$} & 0.47290325 & -1.36384 & {$[14,17]$} & 0.47307308 & -1.39106 \\
{$[15,13]$} & 0.47290516 & -1.36413 & {$[16,17]$} & 0.47307863 & -1.39209 \\
{$[16,15]$} & 0.47294898 & -1.37035 & {$[17,15]$} & 0.47308675 & -1.39369 \\
{$[13,15]$} & 0.47297513 & -1.37499 & {$[16,19]$} & 0.47309052 & -1.39421 \\
{$[16,13]$} & 0.47303007 & -1.38409 & {$[18,15]$} & 0.47310355 & -1.39686 \\
{$[13,16]$} & 0.47303305 & -1.38436 & {$[17,18]$} & 0.47310906 & -1.39788 \\
{$[16,16]$} & 0.47305593 & -1.38800 & {$[15,16]$} & 0.47311001 & -1.39775 \\
{$[14,15]$} & 0.47305793 & -1.38863 & {$[18,18]$} & 0.47311071 & -1.39822 \\
{$[15,17]$} & 0.47306712 & -1.39002 & {$[17,19]$} & 0.47311091 & -1.39826 \\
\hline
\end{tabular}

We looked at approximants for $l \geqslant 9$ and $l-3 \leqslant m \leqslant l+3$. We have tabulated here 20 values which showed best convergence.

These estimates are much more precise than the earlier estimates $\lambda=$ $2.1166 \pm 0.001, \theta=-1.307 \pm 0.006$ and $2 v_{p l}=1.306 \pm 0.010$ using a 25 term series. ${ }^{(5)}$ We can rule out the dimensional reduction conjecture with fair confidence.

Above we presented our estimates using four parameter fits. Method of differential approximants has almost become a standard technique for such analysis. ${ }^{(15)}$ In this case, the generating function has a divergent singularity at $x_{c}$. We tried zeroth order differential approximants, they are listed in Table I. We find a very poor convergence in values of $x_{c}$ and $\theta$. Out of 70 approximants, 15 show spurious singularities (singularities with $\left|x_{c}\right|<0.45$ ). We have listed 20 values which showed best convergence. From these we get, $\lambda=2.1142 \pm 0.002$ and $\theta=-1.39 \pm 0.02$. Clearly the series is not very well behaved. This is reflected in the slow convergence of our series. Also Monte-Carlo generated random spiral trees of sizes 1000 (Fig. 2) suggest that the asymptotic behaviour of the series might set in rather late. Because of poor convergence of differential approximants, we have relied on parameter fits for series analysis in this paper.

\subsection{Monte-Carlo Analysis}

With exact enumeration, we are restricted to clusters of size thirty seven in two dimensions. The main problem is with the extrapolation since the crossover sizes are likely to be large, as the total angle turned by the largest spiral arm about the origin for a spiral tree of size 1000 is about $2 \pi$ only (Fig. 2). This indicates that the crossover value above 
which asymptotic behaviour sets in would be of order $10^{3}$. We tried to study larger spiral trees using MC methods. Monte-Carlo simulation of branched polymers is a challenging problem. Because of branching, most MC algorithms which are good for linear polymers show critical slowing down for branched polymers. For lattice trees there have been some studies using the cut and paste dynamic MC technique. ${ }^{(9)}$ But with spiral constraint, algorithms involving large scale non local moves are not useful. We used an improved version of incomplete enumeration algorithm proposed recently by us. ${ }^{(7)}$ Using it we could study spiral trees of sizes up to one thousand on a square lattice.

Incomplete enumeration is a simple modification of exact enumeration algorithm and can be seen as a percolation process on the genealogical tree of the underlying enumeration problem. The optimal behaviour of the algorithm is achieved when we work around the percolation threshold of the genealogical tree. This algorithm falls in the class of stochastic growth algorithm like PERM. ${ }^{(16)}$ We have shown in, ${ }^{(7)}$ that the asymptotic time to produce an independent sample of $n$ sites for trees and animals grows as $\exp \left(a n^{b}\right)$ with $0<b<1$ for this algorithm. Though the coefficient in front of stretched exponential can be made small by optimising the algorithm for small sizes. We will not give more details of the algorithm in this paper. These can be found in. ${ }^{(7)}$

Figure 2 shows pictures of some typical spiral trees of one thousand sites. Clearly, their structure is very different from lattice trees without the spiral constraint. Because of the constraint they tend to branch much less. For spiral constraint, earlier numerical evidence suggest that unlike lattice trees and animals, spiral trees and animals do not lie in same universality class. The reason is that by allowing loops, the polymer can bend much more often and hence spiral animals would be more compact than the spiral trees.

We studied spiral trees up to sizes 1000 using incomplete enumeration MC method. We made $10^{7} \mathrm{MC}$ runs. The moment of inertia tensor $I_{p l, n}$ as a function of $n$ is plotted in Figs. 7 and 8. Assuming the asymptotic form to be such that

$$
\log \left(I_{p l, n}\right)=\log C+\left(2 v_{p l}+1\right) \log n+\frac{D}{n}
$$

Using above written form, we get the estimate of $v_{p l}$ to be (Figs. 7 and Fig. 8)

$$
2 v_{p l}=1.312 \pm 0.010
$$




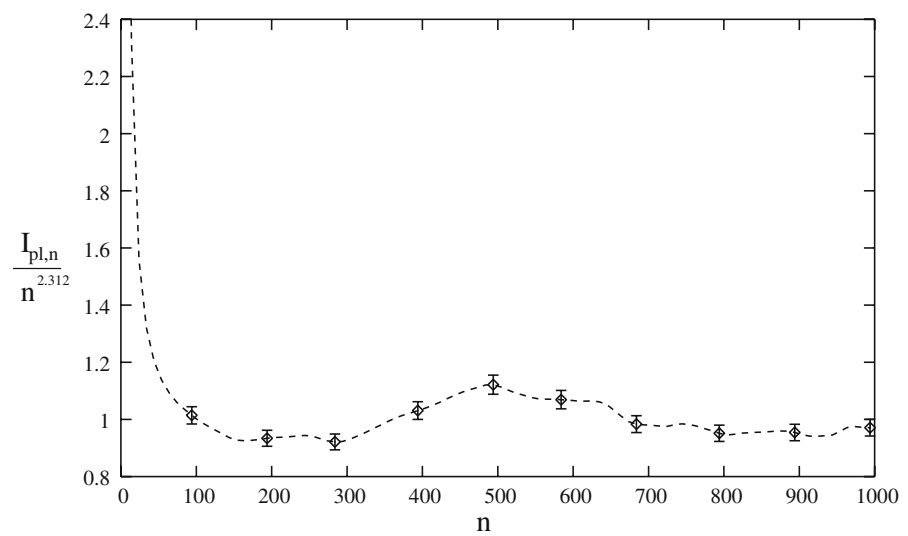

Fig. 7. Plot of $\frac{I_{p l, n}}{n^{2.312}}$ as a function of $n$ for Monte-Carlo generated spiral trees on a square lattice.

In incomplete enumeration MC algorithm, ${ }^{(7)}$ each configuration of $n$ sites is generated with equal probability $P_{n}$ which is just $\prod_{i=1}^{n} p_{i}$, where $p_{i}$ is the probability with which an edge between level $i$ and $i+1$ on the genealogical tree of the problem is chosen. By keeping track of the average number of clusters of a given size generated in a given run, one can estimate the growth constant $\lambda$ and the critical exponent $\theta$. But, the variance of the number of clusters increases as $\exp \left(n^{\alpha}\right), 0<\alpha<1$ for large $n$. Hence, instead we counted the number of descendants of each spiral tree generated. This approach has

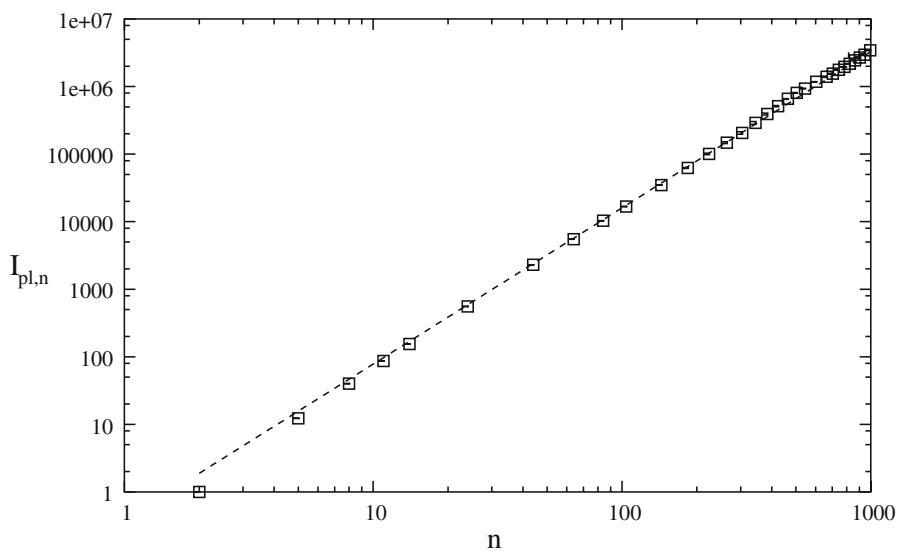

Fig. 8. Plot of $I_{p l, n}$ vs $n$ for Monte-Carlo generated spiral trees on a square lattice. The dotted line is a straight line with slope 2.312 . 


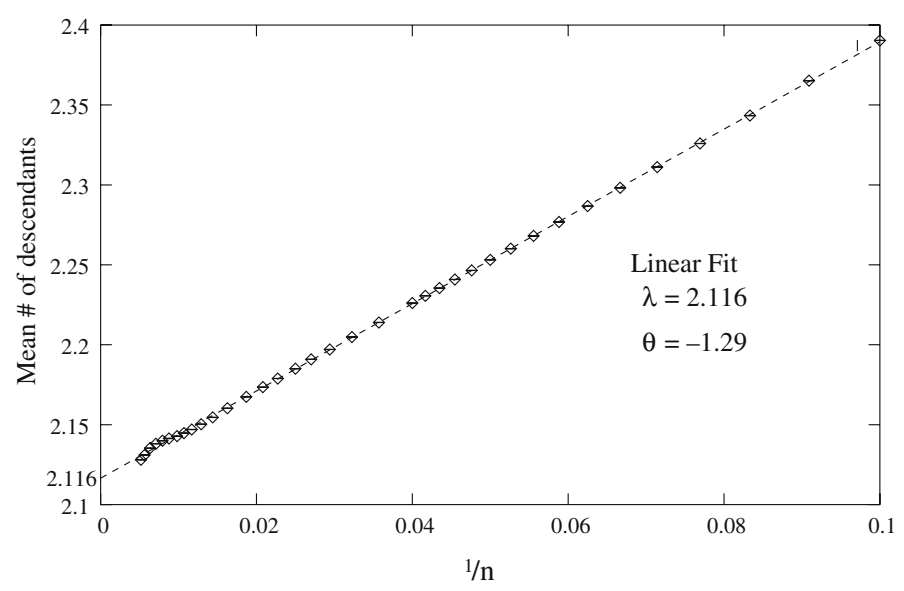

Fig. 9. Monte-Carlo estimates of ratios of the number of configurations on a square lattice. The straight line gives a linear fit of the form $\lambda(1-\theta / n)$ to the data.

been used previously in. ${ }^{(5,17)}$ The mean number of descendants of a tree of size $n$ gives a direct estimate of $A_{n+1} / A_{n}$. We represent the mean number of descendants by $M_{n}$. This is plotted in Fig. 9. A linear fit of the form $\lambda(1-\theta / n)$ to this data gives $\lambda=2.116 \pm 0.01$ and $\theta=-1.29 \pm 0.02$. For better estimates we assume

$$
\log M_{n}=\log \lambda-\theta \log \left(\frac{n+\delta}{n-1+\delta}\right)
$$

With this we get the following estimates for $n \leqslant 200$ which are in agreement with the value obtained by extrapolating the exact series expansions.

$$
\begin{gathered}
\lambda=2.1145 \pm 0.0010 \\
\theta=-1.364 \pm 0.010
\end{gathered}
$$

with $\delta=1.8$.

\section{SPIRAL TREES ON A CUBIC LATTICE}

In dimensions higher than two, the spiral constraint defined as the projection of path joining any site of the tree to the root in $x-y$ plane contains no left turn can be employed in two ways. Bose et al. ${ }^{(5)}$ defined it such that for the projected path from origin to site on $x-y$ plane only forward and right turns are allowed. But in dimensions higher than two, we can define another variation where trees as long as they do not violate 


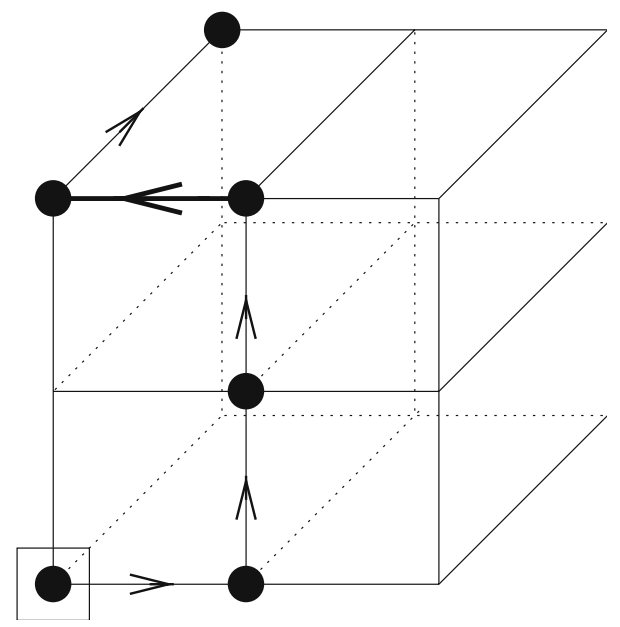

Fig. 10. A spiral tree of six sites on a cubic lattice with a back-turn (drawn by a thicker line). This configuration will contribute to spiral trees $S T_{2}$ of six sites but not to $S T_{1}$.

the tree constraint and the projection on $x-y$ plane is spiral, are allowed. We will call the spiral trees with only forward and right turns allowed as $S T_{1}$.

If we allow for back-turns also, we would get different series because for example, Fig. 10 shows one spiral tree of six sites which would not be a valid configuration if we consider only forward and right turns. We call the spiral trees with back-turns allowed as $S T_{2}$. Naively, one would expect these two to belong to the same universality class. We generated the series till $n=17$ on a cubic lattice using both definitions, however we find the two series behaving differently. Series for both $S T_{1}$ and $S T_{2}$ are given in Appendix A.

For $S T_{1}$, for $A_{n}$ the number of configurations, using Eq. (18) we find that the sequential fit shows a good convergence around $\delta=2.43$. With $\delta=2.43$ and $B=0.094$, the values of $\lambda$ and $\theta$ obtained are listed in Table I. For $v_{p l}$ and $v_{\perp}$, we used fitting form as given in Eq. (21), with $\delta=-1.46$ and $\delta=-0.43$, respectively. The sequential fits are given in Appendix B and estimates are listed in Table II.

Similarly, we obtained 17 term series for $S T_{2}$. The sequential fits are given in Appendix B and the values of $\lambda, \theta, v_{p l}$ and $\nu_{\perp}$ are listed in Table II.

The difference in value of $\lambda$ for $S T_{1}$ and $S T_{2}$ is understandable as $S T_{2}$ has a greater number of configurations. More surprisingly, the critical exponents $\theta, v_{p l}$ and $v_{\perp}$ within our error estimates are different in 
Table II. Estimates of Critical Exponents and Growth Constant from Series Analysis in Three and Four Dimensions

\begin{tabular}{lccrr}
\hline & $S T_{1}(d=3)$ & $S T_{2}(d=3)$ & \multicolumn{1}{c}{$S T_{1}(d=4)$} & \multicolumn{1}{c}{$S T_{2}(d=4)$} \\
\hline$\lambda$ & $5.340 \pm 0.020$ & $5.683 \pm 0.020$ & $9.62 \pm 0.10$ & $10.20 \pm 0.10$ \\
$\theta$ & $-0.675 \pm 0.050$ & $-0.167 \pm 0.050$ & $-0.11 \pm 0.10$ & $0.29 \pm 0.10$ \\
$v_{p l}$ & $0.44 \pm 0.05$ & $0.477 \pm 0.05$ & $0.34 \pm 0.05$ & $0.37 \pm 0.05$ \\
$v_{\perp}$ & $0.54 \pm 0.05$ & $0.69 \pm 0.05$ & $0.44 \pm 0.05$ & $0.45 \pm 0.05$ \\
\hline
\end{tabular}

Note that the value of $\theta$ and $v$ for rooted lattice animals/trees in $3 d$ and $4 d$ is known exactly (in $3 d, \theta=v=1 / 2$ and in $4 d, \theta=5 / 6$ and $\nu=5 / 12$ ).

Table III. Estimates of Critical Exponents and Growth Constants from Monte-Carlo Simulations in Four Dimensions

\begin{tabular}{lcl}
\hline & $S T_{1}(d=4)$ & $S T_{2}(d=4)$ \\
\hline$\lambda$ & $9.60 \pm 0.1$ & $10.2 \pm 0.1$ \\
$\theta$ & $-0.13 \pm 0.1$ & $0.17 \pm 0.1$ \\
$\nu_{p l}$ & $0.33 \pm 0.02$ & $0.38 \pm 0.05$ \\
$\nu_{\perp}$ & $0.451 \pm 0.020$ & $0.455 \pm 0.050$ \\
\hline
\end{tabular}

two models. In neither case, the conjectured dimensional reduction(Eqs. (1) and (2)) seems to be satisfied.

\section{SPIRAL TREES IN FOUR DIMENSIONS}

On a hyper cubic lattice in four dimensions we generated a series till $n=13$. We also correct mistakes in the earlier series reported for $S T_{1}$ in. ${ }^{(5)}$ The corrected series in given in the Appendix A. We also obtained a 13 term series for $S T_{2}$ (see Appendix A). The estimates of $\lambda$ and critical exponents are listed in Table II.

We also performed Monte-Carlo simulations using incomplete enumeration algorithm for spiral trees up to size 50. Our estimates from MC simulations for $S T_{1}$ are given in Table III.

Though we cannot rule out the possibility of $\theta$ being zero in both series analysis and Monte-Carlo simulations, but it seems unlikely. 


\section{DISCUSSION}

Bose et al. gave a plausible argument of curling up of the dimensions in the spiralling plane and had conjectured a dimensional reduction by four for spiral trees. Our numerical evidence as presented in this paper does not support the conjecture. The spiral constraint for trees seems to be very special. For example, the structure of spiral trees is very different from spiral animals with loops allowed. ${ }^{(18)}$ Different implementation of the constraint in $d>2$, seems to give different critical behaviour, suggesting different universality classes. A variety of self avoiding walks with different step restrictions rules on simple cubic lattice were studied in ${ }^{(19)}$ using exact enumeration. Their analysis suggested same universality class for self avoiding walks with various restrictions (including the spiral constraint), as the unrestricted self avoiding walks. In contrast, our studies show different critical behaviour of spiral trees with different geometrical restrictions in three and four dimensions.

We should note that for the large clusters of size $10^{3}$ generated by Monte-Carlo, the total angle turned by the largest spiral arm about the origin is about $2 \pi$. It is possible that the structure of spiral trees is such that this angle tends to infinity as $n$ tends to infinity. In this case the crossover value above which asymptotic behaviour sets in would be expected to be of order $10^{3}$, and series analysis for smaller $n$ may not give correct limiting behaviour. One indication that trees where spiral turns a lot are important is that the growth constant for spiral trees in a quadrant $Q_{1}(x)$ seems to be significantly smaller than for full spiral trees.

For quadrant spiral trees on a square lattice, we obtained exact series up to sizes 56. There are very few such long series known for lattice models. The series gives a estimate of $\lambda=2.044$ for these quadrant spiral trees. This value is significantly smaller than for the full spiral trees.

\section{APPENDIX A}

Exact series enumeration values in different dimensions 
A.1. Two Dimensions

\begin{tabular}{|c|c|c|}
\hline Cluster size $(n)$ & $A_{n}$ & $\left\langle I_{p l, n}\right\rangle$ \\
\hline 1 & 1 & 0 \\
\hline 2 & 4 & 1 \\
\hline 3 & 14 & 3.142857 \\
\hline 4 & 40 & 6.800000 \\
\hline 5 & 105 & 12.266667 \\
\hline 6 & 268 & 19.656716 \\
\hline 7 & 674 & 28.919881 \\
\hline 8 & 1660 & 40.159036 \\
\hline 9 & 4021 & 53.513056 \\
\hline 10 & 9612 & 69.074906 \\
\hline 11 & 22734 & 86.926014 \\
\hline 12 & 53276 & 107.140851 \\
\hline 13 & 123916 & 129.787372 \\
\hline 14 & 286376 & 154.926432 \\
\hline 15 & 658100 & 182.624835 \\
\hline 16 & 1504900 & 212.938547 \\
\hline 17 & 3426464 & 245.919131 \\
\hline 18 & 7771444 & 281.619675 \\
\hline 19 & 17565064 & 320.089299 \\
\hline 20 & 39576360 & 361.374917 \\
\hline 21 & 88916877 & 405.522760 \\
\hline 22 & 199252252 & 452.577078 \\
\hline 23 & 445438310 & 502.580546 \\
\hline 24 & 993616344 & 555.575100 \\
\hline 25 & 2211923712 & 611.601183 \\
\hline 26 & 4914811468 & 670.697934 \\
\hline 27 & 10901498938 & 732.903853 \\
\hline 28 & 24141259980 & 798.256392 \\
\hline 29 & 53379537257 & 866.791847 \\
\hline 30 & 117861710196 & 938.545859 \\
\hline 31 & 259891311248 & 1013.553288 \\
\hline 32 & 572356464452 & 1091.848086 \\
\hline 33 & 1259008971656 & 1173.463504 \\
\hline 34 & 2766351037428 & 1258.432171 \\
\hline 35 & 6071954146120 & 1346.786006 \\
\hline 36 & 13314252070412 & \\
\hline 37 & 29167189621351 & \\
\hline
\end{tabular}


A.2. Three Dimensions $S T_{1}$

\begin{tabular}{rrll}
\hline$n$ & $A_{n}$ & $\left\langle I_{p l, n}\right\rangle$ & $\left\langle I_{\perp, n}\right\rangle$ \\
\hline 1 & 1 & 0. & 0. \\
2 & 6 & 0.66666 & 0.333333 \\
3 & 41 & 1.85366 & 1.07317 \\
4 & 260 & 3.63076 & 2.27692 \\
5 & 1568 & 6.02296 & 3.98214 \\
6 & 9190 & 9.06464 & 6.19913 \\
7 & 53090 & 12.75954 & 8.91987 \\
8 & 303900 & 17.09588 & 12.1405 \\
9 & 1727691 & 22.0606 & 15.8606 \\
10 & 9767426 & 27.6424 & 20.0821 \\
11 & 54966550 & 33.8322 & 24.8071 \\
12 & 308138528 & 40.6214 & 30.0376 \\
13 & 1721739000 & 48.0022 & 35.7754 \\
14 & 9592901762 & 55.9676 & 42.0229 \\
15 & 53314247488 & 64.5112 & 48.7822 \\
16 & 295644339728 & 73.6274 & 56.0556 \\
17 & 1636179620652 & 83.3112 & 63.8454 \\
\hline
\end{tabular}

A.3. Three Dimensions $S T_{2}$

\begin{tabular}{|c|c|c|c|}
\hline$n$ & $A_{n}$ & $\left\langle I_{p l, n}\right\rangle$ & $\left\langle I_{\perp, n}\right\rangle$ \\
\hline 1 & 1 & 0 & 0 . \\
\hline 2 & 6 & 0.666666 & 0.333333 \\
\hline 3 & 41 & 1.85366 & 1.07317 \\
\hline 4 & 260 & 3.63076 & 2.27692 \\
\hline 5 & 1576 & 6.00762 & 4.00761 \\
\hline 6 & 9342 & 9.00192 & 6.30208 \\
\hline 7 & 54890 & 12.60084 & 9.17041 \\
\hline 8 & 320952 & 16.7848 & 12.6182 \\
\hline 9 & 1869907 & 21.5398 & 16.651 \\
\hline 10 & 10861750 & 26.8572 & 21.2772 \\
\hline 11 & 62939998 & 32.7312 & 26.5047 \\
\hline 12 & 364004296 & 39.156 & 32.3409 \\
\hline 13 & 2101795408 & 46.1276 & 38.7927 \\
\hline 14 & 12119643750 & 53.6422 & 45.8667 \\
\hline 15 & 69805866848 & 61.6968 & 53.5693 \\
\hline 16 & 401668709200 & 70.2898 & 61.9068 \\
\hline 17 & 2309283650656 & 79.4192 & 70.8851 \\
\hline
\end{tabular}


A.4. Four Dimensions $S T_{1}$

\begin{tabular}{rrll}
\hline$n$ & $A_{n}$ & $\left\langle I_{p l, n}\right\rangle$ & $\left\langle I_{\perp, n}\right\rangle$ \\
\hline 1 & 1 & 0. & 0 \\
2 & 8 & 0.5 & 0.5 \\
3 & 80 & 1.35 & 1.5 \\
4 & 800 & 2.54 & 3.030 \\
5 & 7912 & 4.05864 & 5.10010 \\
6 & 77656 & 5.89816 & 7.70862 \\
7 & 759172 & 8.04822 & 10.84584 \\
8 & 7403292 & 10.49742 & 14.50268 \\
9 & 72073417 & 13.23410 & 18.67008 \\
10 & 700774524 & 16.24692 & 23.34 \\
11 & 6806914432 & 19.52526 & 28.5052 \\
12 & 66064406668 & 23.0592 & 34.1596 \\
13 & 640741734643 & 26.8396 & 40.2974 \\
\hline
\end{tabular}

A.5. Four dimensions $S T_{2}$

\begin{tabular}{|c|c|c|c|}
\hline$n$ & $A_{n}$ & $\left\langle I_{p l, n}\right\rangle$ & $\left\langle I_{\perp, n}\right\rangle$ \\
\hline 1 & 1 & 0 . & 0 . \\
\hline 2 & 8 & 0.5 & 0.5 \\
\hline 3 & 80 & 1.35 & 1.5 \\
\hline 4 & 800 & 2.54 & 3.030 \\
\hline 5 & 7960 & 4.05226 & 5.10754 \\
\hline 6 & 79048 & 5.87628 & 7.74208 \\
\hline 7 & 785748 & 7.99822 & 10.93174 \\
\hline 8 & 7822676 & 10.40506 & 14.6724 \\
\hline 9 & 78011513 & 13.08484 & 18.95778 \\
\hline 10 & 779189988 & 16.0274 & 23.7816 \\
\hline 11 & 7793590224 & 19.22410 & 29.1376 \\
\hline 12 & 78049539204 & 22.6676 & 35.0206 \\
\hline 13 & 782488672931 & 26.3518 & 41.4252 \\
\hline
\end{tabular}




\section{APPENDIX B}

\section{B.1. Two Dimensions Sequential Fit}

\begin{tabular}{|c|c|c|c|}
\hline$n$ & $\lambda_{n}$ & $\theta_{n}$ & $2 v_{p l, n}$ \\
\hline 5 & 2.078982187 & -1.4143616 & 1.2918751 \\
\hline 6 & 2.118727624 & -1.3598402 & 1.3047319 \\
\hline 7 & 2.117039314 & -1.3623751 & 1.3092198 \\
\hline 8 & 2.115352878 & -1.3651395 & 1.3108492 \\
\hline 9 & 2.114617151 & -1.3664433 & 1.3117861 \\
\hline 10 & 2.114771869 & -1.3661493 & 1.312420 \\
\hline 11 & 2.113813740 & -1.3680905 & 1.3128895 \\
\hline 12 & 2.113978775 & -1.3677359 & 1.3132536 \\
\hline 13 & 2.114183882 & -1.3672706 & 1.3135423 \\
\hline 14 & 2.114099443 & -1.3674721 & 1.3137672 \\
\hline 15 & 2.114103267 & -1.3674625 & 1.3139586 \\
\hline 16 & 2.114205656 & -1.3671946 & 1.3141194 \\
\hline 17 & 2.114223238 & -1.3671466 & 1.3142505 \\
\hline 18 & 2.114256310 & -1.3670527 & 1.3143596 \\
\hline 19 & 2.114279786 & -1.3669834 & 1.3144497 \\
\hline 20 & 2.114291286 & -1.3669483 & 1.3145234 \\
\hline 21 & 2.114301033 & -1.3669174 & 1.3145839 \\
\hline 22 & 2.114310834 & -1.3668854 & 1.3146334 \\
\hline 23 & 2.114311487 & -1.3668832 & 1.3146734 \\
\hline 24 & 2.114314464 & -1.3668728 & 1.3147059 \\
\hline 25 & 2.114318963 & -1.3668566 & 1.3147321 \\
\hline 26 & 2.114320428 & -1.3668513 & 1.3147529 \\
\hline 27 & 2.114321722 & -1.3668464 & 1.3147694 \\
\hline 28 & 2.114324605 & -1.3668351 & 1.3147823 \\
\hline 29 & 2.114326551 & -1.3668274 & 1.3147921 \\
\hline 30 & 2.114327932 & -1.3668217 & 1.3147994 \\
\hline 31 & 2.114329734 & -1.3668142 & 1.3148047 \\
\hline 32 & 2.114331349 & -1.3668072 & 1.3148083 \\
\hline 33 & 2.114332328 & -1.3668029 & 1.3148104 \\
\hline 34 & 2.114333055 & -1.3667997 & 1.3148113 \\
\hline 35 & 2.114333550 & -1.3667974 & 1.3148113 \\
\hline 36 & 2.114333553 & -1.3667974 & \\
\hline Es. Val. & $2.11433 \pm 0.0001$ & $-1.3667 \pm 0.001$ & $1.3148 \pm 0.001$ \\
\hline
\end{tabular}


B.2. Three Dimensions $S T_{2}$

\begin{tabular}{ccccc}
\hline$n$ & $\lambda_{n}$ & $\theta_{n}$ & $2 v_{p l, n}$ & $2 v_{\perp, n}$ \\
\hline 5 & 5.153269 & -1.019107 & 0.847865 & 1.128949 \\
6 & 5.275382 & -0.810187 & 0.865641 & 1.098419 \\
7 & 5.310873 & -0.743662 & 0.871330 & 1.083814 \\
8 & 5.319667 & -0.725590 & 0.874191 & 1.077873 \\
9 & 5.327658 & -0.707695 & 0.875922 & 1.073809 \\
10 & 5.334141 & -0.691977 & 0.876525 & 1.070550 \\
11 & 5.337903 & -0.682161 & 0.876502 & 1.068326 \\
12 & 5.339533 & -0.677605 & 0.876303 & 1.067085 \\
13 & 5.340111 & -0.675880 & 0.876176 & 1.066526 \\
14 & 5.340282 & -0.675339 & 0.876139 & 1.066334 \\
15 & 5.340255 & -0.675428 & 0.876197 & 1.066365 \\
Es. Val. & $5.340 \pm 0.02$ & $-0.675 \pm 0.05$ & $0.876 \pm 0.05$ & $1.066 \pm 0.05$ \\
\hline
\end{tabular}

B.3. Three Dimensions $S T_{2}$

\begin{tabular}{ccccc}
\hline$n$ & $\lambda_{n}$ & $\theta_{n}$ & $2 v_{p l, n}$ & $2 v_{\perp, n}$ \\
\hline 5 & 5.694072 & -0.143802 & 1.054721 & 1.200021 \\
6 & 5.719085 & -0.123718 & 1.013833 & 1.171160 \\
7 & 5.710159 & -0.132441 & 0.989310 & 1.153823 \\
8 & 5.695471 & -0.149408 & 0.977105 & 1.147515 \\
9 & 5.689143 & -0.157845 & 0.969817 & 1.144294 \\
10 & 5.687350 & -0.160552 & 0.963977 & 1.141637 \\
11 & 5.686110 & -0.162645 & 0.959561 & 1.139686 \\
12 & 5.684763 & -0.165153 & 0.956738 & 1.138646 \\
13 & 5.683809 & -0.167099 & 0.955250 & 1.138243 \\
14 & 5.683473 & -0.167843 & 0.954653 & 1.138124 \\
15 & 5.683632 & -0.167463 & 0.954662 & 1.138134 \\
Es. Val. & $5.683 \pm 0.02$ & $-0.167 \pm 0.05$ & $0.954 \pm 0.05$ & $1.138 \pm 0.05$ \\
\hline
\end{tabular}




\section{ACKNOWLEDGMENTS}

I am thankful to my adviser Prof. Deepak Dhar for suggesting the problem, for discussions and for useful comments on the manuscript.

\section{REFERENCES}

1. I. Hargittai and C. A. Pickover, eds., Spiral Symmetry (World Scientific, Singapore, 1992).

2. V. Privman, Spiral self-avoiding walks, J. Phys. A: Math. Gen. 16:L571 (1983).

3. A. J. Guttmann and N. C. Wormald, On the number of spiral self-avoiding walks, J. Phys. A: Math. Gen. 17:271 (1984).

4. T. C. Li and Z. C. Zhou, Spiral bond animals-ratio approach, J. Phys. A: Math. Gen. 18:67 (1985).

5. I. Bose, P. Ray and D. Dhar, Rooted spiral trees in dimensions 2, 3 and 4, J. Phys. A: Math. Gen. 21:L219 (1988).

6. D. C. Brydges and J. Z. Imbrie, Branched polymers and dimensional reduction, Ann. Math. 158:1019 (2003).

7. Sumedha and D. Dhar, Efficiency of the incomplete enumeration algorithm for MonteCarlo simulation of linear and branched polymers, cond-mat/0408640, J. Stat. Phys., accepted for publication.

8. D. J. Klein, Rigorous results for branched polymers with excluded volume, J. Chem. Phys. 75:5186 (1981).

9. N. Madras, A rigorous bound on the critical exponent for the number of lattice trees, animals and polygons, J. Stat. Phys. 78:681 (1995).

10. I. Jensen, Enumerations of lattice animals and trees, J. Stat. Phys. 102:865 (2001).

11. J. W. Essam, Percolation processes. II The pair connectedness, J. Math. Phys. 12:874 (1971).

12. J. L. Martin, in Computer Techniques for Evaluating Lattice Constants, Phase Transitions and Critical Phenomena, Domb and Green, eds., Vol.3 (Academic Press London, 1983).

13. I. G. Enting, Generating functions for enumerating self-avoiding rings on the square lattice, J. Phys. A: Math. Gen. 13:3713 (1980).

14. A. Conway, Enumerating $2 D$ percolation series by finite-lattice method: theory $J$. Phys. A: Math. Gen. 28:335 (1995).

15. D. S. Gaunt and A. J. Guttmann, in Asymptotic Analysis of Coeffcients, Phase Transitions and Critical Phenomena, Domb and Green, eds., Vol. 3 (Academic Press London, 1983).

16. P. Grassberger and W. Nadler, Go with the winners - Simulations, cond-mat/0010265, Proceedings der Heraeus-Ferienschule "Vom Billiardtisch bis Monte Carlo: Spielfelder der statistischen Physik", Chemnitz, October 2000.

17. E. J. Janse van Rensburg and A. Rechnitzer, High Precision canonical Monte Carlo determination of the growth constant of square lattice trees, Phys. Rev. E 67:036116 (2003).

18. I. Bose, in Lattice Animals and the Percolation Model under Rotational Constraint, Percolation Theory and Particle Systems, Rahul Roy, ed. (University Press, India, 2000).

19. A. Rechnitzer and A. L. Owczarek, On three-dimensional self-avoiding walk symmetry classes J. Phys. A:Math. Gen. 33:2685 (2000). 